LAWRENCE LIVERMORE N A T IO N A L LABORATORY
Ab Initio No-Core Shell Model Calculations Using Realistic Two- and Three-Body Interactions

P. Navratil, W. E. Ormand, C. Forssen, E. Caurier

December 1, 2004

ENAM 04: The Fourth International Conference on Exotic Nuclei and Atomic Masses

Callaway Gardens, Pine Mountain, GA, United States September 12, 2004 through September 16, 2004 
This document was prepared as an account of work sponsored by an agency of the United States Government. Neither the United States Government nor the University of California nor any of their employees, makes any warranty, express or implied, or assumes any legal liability or responsibility for the accuracy, completeness, or usefulness of any information, apparatus, product, or process disclosed, or represents that its use would not infringe privately owned rights. Reference herein to any specific commercial product, process, or service by trade name, trademark, manufacturer, or otherwise, does not necessarily constitute or imply its endorsement, recommendation, or favoring by the United States Government or the University of California. The views and opinions of authors expressed herein do not necessarily state or reflect those of the United States Government or the University of California, and shall not be used for advertising or product endorsement purposes. 


\title{
Ab Initio No-Core Shell Model Calculations Using Realistic Two- and Three-Body Interactions *
}

\author{
P. Navrátil ${ }^{1}$, W. E. Ormand ${ }^{1}$, C. Forssén ${ }^{1}$ and E. Caurier ${ }^{2}$ \\ 1 Lawrence Livermore National Laboratory, L-414, P.O. Box 808, Livermore, CA 94551, USA \\ ${ }^{2}$ Institut de Recherches Subatomiques, IN2P3-CNRS, Universite Louis Pasteur, F-67037 Strasbourg, France
}

Received: date / Revised version: date

\begin{abstract}
There has been significant progress in the $a b$ initio approaches to the structure of light nuclei. One such method is the ab initio no-core shell model (NCSM). Starting from realistic two- and threenucleon interactions this method can predict low-lying levels in $p$-shell nuclei. In this contribution, we present a brief overview of the NCSM with examples of recent applications. We highlight our study of the parity inversion in ${ }^{11} \mathrm{Be}$, for which calculations were performed in basis spaces up to $9 \hbar \Omega$ (dimensions reaching $7 \times 10^{8}$ ). We also present our latest results for the $p$-shell nuclei using the Tucson-Melbourne $\mathrm{TM}^{\prime}$ three-nucleon interaction with several proposed parameter sets.
\end{abstract}

PACS. 21.60.-n Nuclear structure models and methods - 21.30.Fe Forces in hadronic systems and effective interactions

\section{Introduction}

In recent years, construction of accurate nucleon-nucleon potentials and increases in computing power have led to new methods capable of solving the nuclear structure problem for systems of more than four nucleons $[1,2]$. One such method is the $a b$ initio no-core shell model (NCSM) [2]. The principal foundation of this approach is the use of effective interactions appropriate for the large, but finite, basis spaces employed in the calculations. These effective interactions are derived from the underlying realistic internucleon potentials by a unitary transformation in a way that guarantees convergence to the exact solution as the basis size increases.

In this contribution, we briefly discuss the NCSM theory, present a convergence test of the method as well as selected nuclear structure results for light nuclei up to $A=13$. We highlight our recent study of the parity inversion in ${ }^{11} \mathrm{Be}$, for which calculations were performed using several modern nucleon-nucleon potentials in basis spaces up to $9 \hbar \Omega$ (dimensions reaching $7 \times 10^{8}$ ).

At present, the $a b$ initio NCSM is capable of including the much-less-explored genuine three-nucleon forces $[3,4]$. An important result of these nuclear-structure studies is the significance of the three-nucleon interaction in

\footnotetext{
* This work was performed in part under the auspices of the U. S. Department of Energy by the University of California, Lawrence Livermore National Laboratory under contract No. W-7405-Eng-48. Support from the LDRD contract No. 04ERD-058, and from U.S. Department of Energy, Office of Science, (Work Proposal Number SCW0498) is acknowledged.
}

determining not only the binding energy, but also the excitation spectra and other observables. Consequently, nuclear-structure calculations are becoming a tool in discriminating different three-body interaction models and at the same time can put constraints on the three-body force parameters. As a step in this direction, we have improved the accuracy of our three-body interaction calculations and obtained results for the $p$-shell nuclei using the Tucson- Melbourne TM $^{\prime}$ three-nucleon interaction [5] with several proposed parameter sets [6].

\section{$2 \mathrm{Ab}$ initio no-core shell model}

We consider a system of $A$ point-like nonrelativistic nucleons that interact by realistic two- or two- plus threenucleon interactions. As the simpler case, when just the two-nucleon interaction is considered, was discussed in several papers, see e.g. Ref. [2], we focus here on the more general case when both two- and three-nucleon interactions (TNI) are included. The starting Hamiltonian is then

$$
\begin{aligned}
H_{A}= & \frac{1}{A} \sum_{i<j} \frac{\left(\boldsymbol{p}_{i}-\boldsymbol{p}_{j}\right)^{2}}{2 m} \\
& +\sum_{i<j}^{A} V_{\mathrm{NN}, i j}+\sum_{i<j<k}^{A} V_{\mathrm{NNN}, i j k}
\end{aligned}
$$

where $m$ is the nucleon mass, $V_{\mathrm{NN}, i j}$ is the nucleon-nucleon $(\mathrm{NN})$ interaction, and $V_{\mathrm{NNN}, i j k}$ is the three-nucleon interaction. In the NCSM, we employ a large but finite 
harmonic-oscillator (HO) basis. Due to properties of the realistic nuclear interaction in Eq. (1), we must derive an effective interaction appropriate for the basis truncation. To facilitate the derivation of the effective interaction, we modify the Hamiltonian (1) by adding to it the center-ofmass (CM) HO Hamiltonian $H_{\mathrm{CM}}=T_{\mathrm{CM}}+U_{\mathrm{CM}}$, where $U_{\mathrm{CM}}=\frac{1}{2} A m \Omega^{2} \boldsymbol{R}^{2}, \boldsymbol{R}=\frac{1}{A} \sum_{i=1}^{A} \boldsymbol{r}_{i}$. The effect of the HO CM Hamiltonian will later be subtracted out in the final many-body calculation. Due to the translational invariance of the Hamiltonian (1) the HO CM Hamiltonian has in fact no effect on the intrinsic properties of the system in the infinite basis space. The modified Hamiltonian can be cast into the form

$$
\begin{aligned}
H_{A}^{\Omega}= & H_{A}+H_{\mathrm{CM}}=\sum_{i=1}^{A} h_{i}+\sum_{i<j}^{A} V_{i j}^{\Omega, A}+\sum_{i<j<k}^{A} V_{\mathrm{NNN}, i j k} \\
= & \sum_{i=1}^{A}\left[\frac{\boldsymbol{p}_{i}^{2}}{2 m}+\frac{1}{2} m \Omega^{2} \boldsymbol{r}_{i}^{2}\right] \\
& +\sum_{i<j}^{A}\left[V_{\mathrm{NN}, i j}-\frac{m \Omega^{2}}{2 A}\left(\boldsymbol{r}_{i}-\boldsymbol{r}_{j}\right)^{2}\right] \\
& +\sum_{i<j<k}^{A} V_{\mathrm{NNN}, i j k} .
\end{aligned}
$$

Next we divide the $A$-nucleon infinite HO basis space into the finite active space $(P)$ comprising of all states of up to $N_{\max } \mathrm{HO}$ excitations above the unperturbed ground state and the excluded space $(Q=1-P)$. The basic idea of the NCSM approach is to apply a unitary transformation on the Hamiltonian (2), $e^{-S} H_{A}^{\Omega} e^{S}$ such that $Q e^{-S} H_{A}^{\Omega} e^{S} P=0$. If such a transformation is found, the effective Hamiltonian that exactly reproduces a subset of eigenstates of the full space Hamiltonian is given by $H_{\text {eff }}=P e^{-S} H_{A}^{\Omega} e^{S} P$. This effective Hamiltonian contains up to $A$-body terms and to construct it is essentially as difficult as to solve the full problem. Therefore, we apply this basic idea on a sub-cluster level. When a genuine TNI is considered, the simplest approximation is to use a three-body effective interaction. The NCSM calculation is then performed with the following four steps:

(i) We solve a three-nucleon system for all possible three-nucleon channels with the Hamiltonian $H_{A}^{\Omega}$,i.e., using $h_{1}+h_{2}+h_{3}+V_{12}^{\Omega, A}+V_{13}^{\Omega, A}+V_{23}^{\Omega, A}+V_{\mathrm{NNN}, 123}$. It is necessary to separate the three-body effective interaction contributions from the TNI and from the two-nucleon interaction. Therefore, we need to find three-nucleon solutions for the Hamiltonian with and without the $V_{\mathrm{NNN}, 123}$ TNI term. The three-nucleon solutions are obtained by procedures described in Refs. [7] (without TNI) and [8] (with TNI). We note that we made some improvements and simplifications to the precedure described in Ref. [8], which allowed us to reach a larger basis size and, consequently, lead to an improved accuracy of our results.

(ii) We construct the unitary transformation corresponding to the choice of the active basis space $P$ from the three-nucleon solutions using the Lee-Suzuki procedure [9, $10]$. (iii) As the three-body effective interactions are derived in the Jacobi-coordinate $\mathrm{HO}$ basis but the $A$-nucleon calculations will be performed in a Cartesian-coordinate single-particle Slater-determinant m-scheme basis, we need to perform a suitable transformation of the interactions. This transformation is a generalization of the well-known transformation on the two-body level that depends on $\mathrm{HO}$ Brody-Moshinsky brackets.

(iv) We solve the Schrödinger equation for the $A \mathrm{nu}-$ cleon system using the Hamiltonian $H_{A, \text { eff }}^{\Omega}=\sum_{i=1}^{A} h_{i}+$ $\frac{1}{A-2} \sum_{i<j<k}^{A} V_{3 \mathrm{eff}, i j k}^{\mathrm{NN}}+\sum_{i<j<k}^{A} V_{3 \mathrm{eff}, i j k}^{\mathrm{NN}}$, where the $\frac{1}{A-2} \mathrm{fac}-$ tor takes care of overcounting the contribution from the two-nucleon interaction. At this point we also subtract the $H_{\mathrm{CM}}$. The $A$ nucleon calculation is then performed using a shell model code generalized to handle three-body interactions.

\section{Convergence test: ${ }^{4} \mathrm{He}$ with the CD-Bonn 2000}

By construction, the ab initio NCSM calculation will converge to the exact result of the starting Hamiltonian with the increase of the model space, $P \rightarrow 1$, that is with $N_{\max } \rightarrow \infty$. Obviously, the idea is that the use of the effective interaction will speed up the convergence significantly compared to a calculation with the starting bare Hamiltonian. Consequently, the hope is that converged results can be obtained with model spaces that are reachable with present computers.

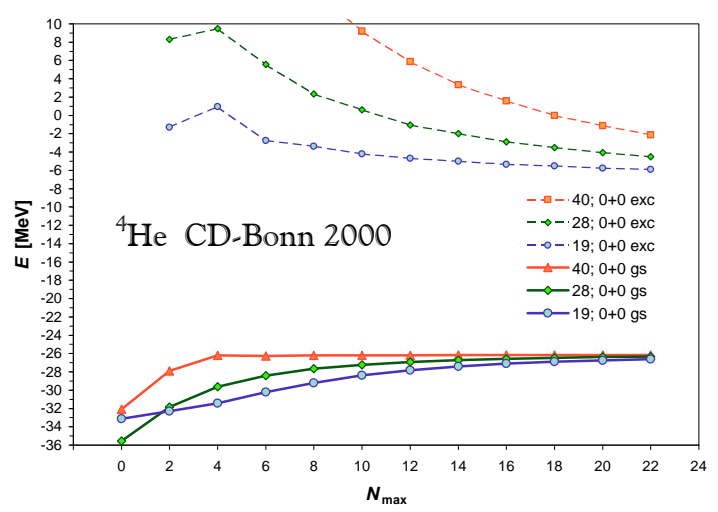

Fig. 1. Ground state and the first $0^{+} 0$ excited state energy of ${ }^{4} \mathrm{He}$ calculated using the CD-Bonn $2000 \mathrm{NN}$ potential. Dependence on the NCSM model-space size $N_{\max }$ for three different $\mathrm{HO}$ frequencies, $\hbar \Omega=19,28$ and $40 \mathrm{MeV}$, is presented.

An example of a succcesfully converged NCSM calculation is shown in Fig. 1. We present the dependence of the ${ }^{4} \mathrm{He}$ ground state and the first $0^{+} 0$ excited state energy on the model space size, defined by $N_{\max }$, for three different HO frequencies. The CD-Bonn $2000 \mathrm{NN}$ potential was used [11]. The calculations were performed with the no-core version of the shell-model code Antoine [12]. 
We observe a fast convergence for the ground state for all three frequencies, with the final result $-26.15(10) \mathrm{MeV}$ in good agreement with a Faddeev-Yakubovsky calculation [13]. The excited state convergence is slower with a stronger frequency dependence due to the more complex structure of this state. Still, we are able to extrapolate the excitation energy to -6.0(3) MeV. Although the CD-Bonn $2000 \mathrm{NN}$ potential underbinds ${ }^{4} \mathrm{He}$ by about $2 \mathrm{MeV}$, the $0_{2}^{+} 0$ excitation energy is described rather well. It is interesting to note that the convergence rate for the ground state is the fastest for the highest HO frequency while the convergence rate for the excited state is the fastest for the lowest HO frequency employed in our presented calculations. Clearly, the optimal frequency for convergence is state dependent and correlated with the radius of the state, the smaller the radius the higher optimal frequency.

\section{Natural- vs. unnatural-parity states in ${ }^{11} \mathrm{Be}$}

Studies on light neutron-rich nuclei has attracted an increasing amount of theoretical and experimental effort ever since the advent of radioactive nuclear beams. One reason for this is the fact that substantial deviations from regular shell structure has been observed in these fewbody systems. The $A=11$ isobar is of particular interest in this respect since it exhibits some anomalous features that are not easily explained in a simple shell-model framework. Most importantly, the parity-inverted $1 / 2^{+}$ground state of ${ }^{11} \mathrm{Be}$ was noticed by Talmi and Unna [14] already in the early 1960s, and it still remains one of the best examples of the disappearance of the $N=8$ magic number. The ability to explain this level inversion within a microscopic theory, such as the ab initio NCSM, is a true challenge of our understanding of nuclear forces. Unfortunately, a shortcoming of the NCSM method is the fact that the HO basis functions have incorrect asymptotics. This might be a problem when trying to describe loosely-bound systems. Therefore it is desirable to include as many terms as possible in the expansion of the total wave function. By restricting ourselves to the use of $\mathrm{NN}$ interactions, we are able to maximize the model space and to better observe the convergence of our results. In order to study the level ordering in ${ }^{11} \mathrm{Be}$, and in particular the relative position of natural- and unnatural-parity states, we have performed large-basis ab initio NCSM calculations using four different high-precision NN interactions [15]. One of these, the non-local INOY interaction [16], has never before been used in nuclear-structure calculations. Although it is formally a two-body potential, it does reproduce the binding energies of ${ }^{3} \mathrm{H}$ and ${ }^{3} \mathrm{He}$ and, to some extent, $3 \mathrm{~N}$ scattering data. Remember that the underbinding of $A>2$ systems is a deficiency of all other realistic NN interactions. However, this achievement comes with the cost of having to sacrifice some of the accuracy of the fit to NN scattering data. In particular, the ${ }^{3} P$ interactions are slightly modified in the IS-M version of the potential that we are using.

In Fig. 2 we show the excitation spectrum for ${ }^{11} \mathrm{Be}$ calculated using the INOY interaction. We were able to reach the $9 \hbar \Omega$ model space, which corresponds to a matrix with dimension exceeding $7 \times 10^{8}$. Although we do not reproduce the anomalous $1 / 2^{+}$ground state with any of the NN interactions being used, we do observe a dramatic drop of the positive-parity excitation energies with increasing model space. This observation is particularly prominent for the INOY results, shown in Fig. 2; which in turn suggests that a realistic $3 \mathrm{~N}$ force will be needed in order to reproduce the parity inversion in microscopic approaches.

In this study we observe a remarkable agreement between the predictions of different standard high-precision $\mathrm{NN}$ interactions. In particular, the relative level spacings observed when plotting positive- and negative-parity states separately, were found to be very stable. The INOY interaction gives a larger binding energy and a stronger spinorbit splitting than the other NN interactions. Note that both these effects would be expected from a genuine TNI, but with INOY they are achieved by the use of shortrange, non-local terms in the $\mathrm{NN}$ interaction.

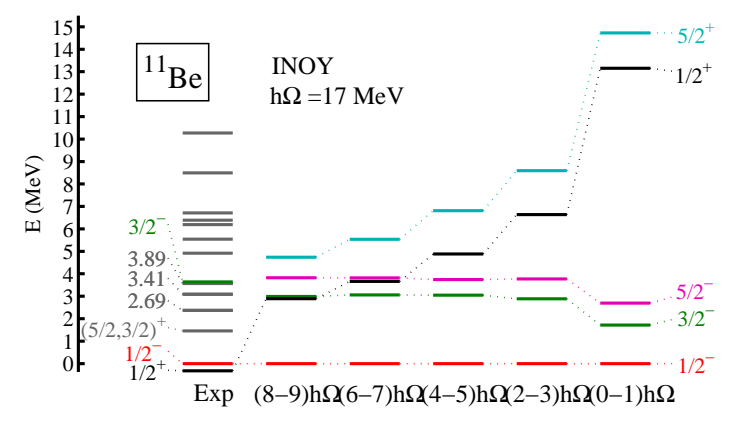

Fig. 2. Excitation spectrum for ${ }^{11} \mathrm{Be}$ calculated using the INOY interaction in $0 \hbar \Omega-9 \hbar \Omega$ model spaces with a fixed $\mathrm{HO}$ frequency of $\hbar \Omega=17 \mathrm{MeV}$. The experimental values are from Ref. [17].

\section{Results with a genuine three-nucleon interaction}

It is well established that standard accurate NN potentials, like AV8'[1] or CD-Bonn 2000 [11], must be augmented by realistic three-body interactions in order to reproduce experimental binding energies, scattering observables and nuclear structure of $A>2$ nuclei.

An interesting example which demonstrates the importance of the TNI is the ground-state spin inversion in ${ }^{10} \mathrm{~B}$. The ground state of ${ }^{10} \mathrm{~B}$ is $3^{+} 0$. Calculations with standard accurate NN potentials, however, predict a $1^{+} 0$ ground state $[1,18,19]$. By including the Tucson-Melbourne $\mathrm{TM}^{\prime}$ TNI, the problem is resolved, see Fig. 3 . In the figure, three parameter sets denoted as 81, 93 and 99 [6] are considered for the TM' TNI. All give similar results, but dramatically different compared to the calculation with only the two-nucleon potential. 


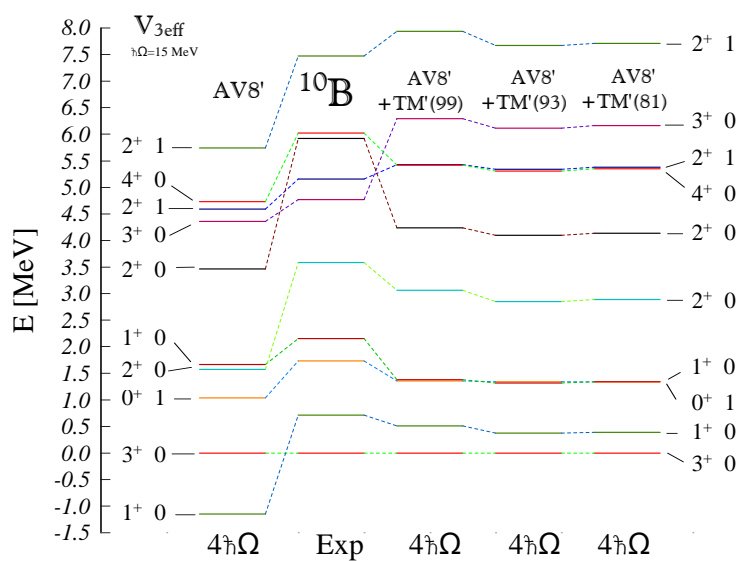

Fig. 3. Excitation spectra of ${ }^{10} B$ obtained using the $\mathrm{AV} 8^{\prime}$ $\mathrm{NN}$ interaction and $\mathrm{AV}^{\prime}+\mathrm{TM}^{\prime}$ interactions, respectively, are compared to experiment. Three parameter sets denoted as 81 , 93 and 99 are considered for the $\mathrm{TM}^{\prime}$ TNI. The $4 \hbar \Omega$ basis space with $\hbar \Omega=15 \mathrm{MeV}$ HO frequency were employed.

In ${ }^{13} \mathrm{C}$, a less dramatic but still significant effect of the TNI is apparent in the excitation spectra shown in Fig. 4. A calculation with the two-nucleon interaction underestimates the level splittings of $\frac{3}{2}^{-}-\frac{1}{2}^{-}$and $\frac{5}{2}_{1}^{-}-\frac{1}{2}^{-}$. Including the $\mathrm{TM}^{\prime} \mathrm{TNI}$ significantly improves agreement of these level splittings with experiment. We can also see a higher sensitivity of the excitation energies to the choice of the TNI parameter set compared to ${ }^{10} \mathrm{~B}$. Contrary to the two-nucleon interaction, the form and parameters of the TNI are much less established. A sensitivity of nuclear structure to the form and parameters of the TNI can then be helpful in determining the fine details of the TNI itself. Obviously, before any conclusions can be drawn, the convergence of the nuclear structure results must be verified. Concerning the NCSM calculations with a two-nucleon interaction, we were able to reach the $10 \hbar \Omega$ and $8 \hbar \Omega$ model spaces for ${ }^{10} \mathrm{~B}$ and ${ }^{13} \mathrm{C}$, respectively. This was sufficient for convergence of excitation energies of low-lying levels. Unfortunately, the NCSM calculations with the TNI are much more involved. Currently, we are limited to $4 \hbar \Omega$ model spaces for the $A=9-16$ nuclei. It is imperative to increase the basis to at least $6 \hbar \Omega$ in order to establish convergence of the excitation energies.

The effects of the TNI in $p$-shell nuclei are not only limited to an increase of binding energies and changes in excitation spectra. In the $A=10-13$ region, we also observe significant TNI influence on the Gamow-Teller and B(M1) transitions. An intresting example is the $0^{+} 0 \rightarrow 1^{+} 1$ transition in $A=12$ [3] known to be highly sensitive to the strength of the spin-orbit force. An improved description of this transition with the TNI demonstrates that the TNI, here in particular the TM'(99), increases the spin-orbit force strength. Similarly, we observe significant TNI effects for Gamow-Teller transistions in ${ }^{11} \mathrm{~B} \rightarrow{ }^{11} \mathrm{C}[4]$ and in transitions from the ${ }^{13} \mathrm{C}$ ground state to low-lying excited states in ${ }^{13} \mathrm{~N}$. Concerning the ${ }^{11} \mathrm{~B} \rightarrow{ }^{11} \mathrm{C}$ transitions, recent

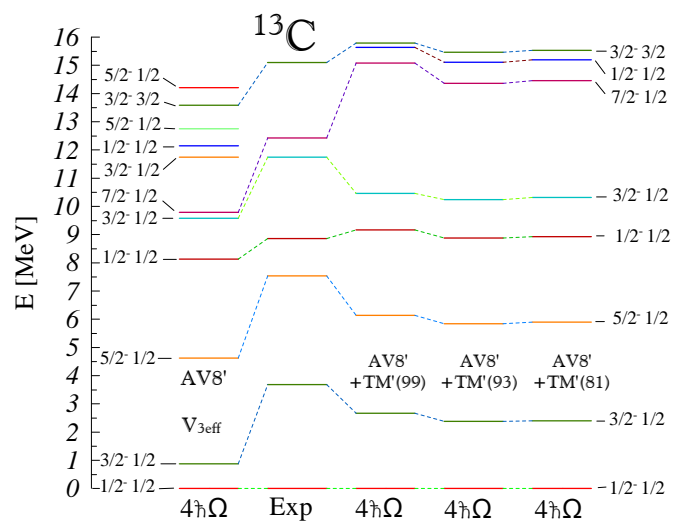

Fig. 4. Excitation spectra of ${ }^{13} C$ obtained using the $\mathrm{AV} 8^{\prime}$ $\mathrm{NN}$ interactions and $\mathrm{AV} 8^{\prime}+\mathrm{TM}^{\prime}$ interactions, respectively, are compared to experiment. Three parameter sets denoted as 81 , 93 and 99 are considered for the $\mathrm{TM}^{\prime}$ TNI. The $4 \hbar \Omega$ basis space and the $\hbar \Omega=15 \mathrm{MeV} H O$ frequency were employed.

experimental data agree much better with our calculated results when the TNI is included [20].

\section{References}

1. B. S. Pudliner et al., Phys. Rev. C 56 (1997) 1720; R. B. Wiringa, Nucl. Phys. A 631 (1998) 70c; R. B. Wiringa et al., Phys. Rev. C 62 (2000) 014001; S. C. Pieper et al., Phys. Rev. C 64 (2001) 014001.

2. P. Navrátil, J. P. Vary and B. R. Barrett, Phys. Rev. Lett. 84, (2000) 5728; Phys. Rev. C 62, (2000) 054311.

3. A. C. Hayes, P. Navrátil and J. P. Vary, Phys. Rev. Lett. 91 (2003) 012502.

4. P. Navrátil and W. E. Ormand, Phys. Rev. C 68 (2003) 034305.

5. S. A. Coon et al., Nucl. Phys. A317 (1979) 242.

6. S. A. Coon and H. K. Han, Few-Body Syst. 30 (2001) 131.

7. P. Navrátil, G. P. Kamuntavičius and B. R. Barrett, Phys. Rev. C 61 (2000) 044001.

8. D. C. J. Marsden, P. Navrátil, S. A. Coon and B. R. Barrett, Phys. Rev. C 66 (2002) 044007.

9. K. Suzuki and S. Y. Lee, Prog. Theor. Phys. 64 (1980) 2091.

10. K. Suzuki and R. Okamoto, Prog. Theor. Phys. 92 (1994) 1045 .

11. R. Machleidt, Phys. Rev. C 63 (2001) 024001.

12. E. Caurier and F. Nowacki, Acta Physica Polonica 30, 705 (1999).

13. A. Nogga, private communication.

14. I. Talmi and I. Unna, Phys. Rev. Lett. 4 (1960) 469.

15. C. Forssén, P. Navrátil, W. E. Ormand and E. Caurier, in preparation.

16. P. Doleschall, Phys. Rev. C 69 (2004) 054001.

17. F. Ajzenberg-Selove, Nucl. Phys. A 5061 (1990) 1, (April 2004 revised manuscript).

18. P. Navrátil and W. E. Ormand, Phys. Rev. Lett. 88 (2002) 152502.

19. E. Caurier, P. Navrátil, W. E. Ormand and J. P. Vary, Phys. Rev. C 66 (2002) 024314.

20. Y. Fujita et al., Phys. Rev. C 70 (2004) 011306(R). 